\title{
Article
}

\section{Binary Operations in Metric Spaces Satisfying Side Inequalities}

\author{
María A. Navascués ${ }^{1, *,+}$ (D), Pasupathi Rajan ${ }^{2,+}$ and Arya Kumar Bedabrata Chand $2,+$ (D) \\ 1 Departamento de Matemática Aplicada, Escuela de Ingeniería y Arquitectura, Universidad de Zaragoza, \\ 50018 Zaragoza, Spain \\ 2 Department of Mathematics, Indian Institute of Technology Madras, Chennai 600036, India; \\ pasupathi4074@gmail.com (P.R.); chand@iitm.ac.in (A.K.B.C.) \\ * Correspondence: manavas@unizar.es; Tel.: +34-97-676-1980 \\ + These authors contributed equally to this work.
}

check for

updates

Citation: Navascués, M.A.

Pasupathi, R.; Chand, A.K.B. Binary

Operations in Metric Spaces

Satisfying Side Inequalities.

Mathematics 2022, 10, 11. https://

doi.org/10.3390/math10010011

Academic Editor: Vasileios

Drakopoulos

Received: 2 November 2021

Accepted: 17 December 2021

Published: 21 December 2021

Publisher's Note: MDPI stays neutral with regard to jurisdictional claims in published maps and institutional affiliations.

Copyright: (C) 2021 by the authors Licensee MDPI, Basel, Switzerland. This article is an open access article distributed under the terms and conditions of the Creative Commons Attribution (CC BY) license (https:// creativecommons.org/licenses/by/ $4.0 /)$.

\begin{abstract}
The theory of metric spaces is a convenient and very powerful way of examining the behavior of numerous mathematical models. In a previous paper, a new operation between functions on a compact real interval called fractal convolution has been introduced. The construction was done in the framework of iterated function systems and fractal theory. In this article we extract the main features of this association, and consider binary operations in metric spaces satisfying properties as idempotency and inequalities related to the distance between operated elements with the same right or left factor (side inequalities). Important examples are the logical disjunction and conjunction in the set of integers modulo 2 and the union of compact sets, besides the aforementioned fractal convolution. The operations described are called in the present paper convolutions of two elements of a metric space $E$. We deduce several properties of these associations, coming from the considered initial conditions. Thereafter, we define self-operators (maps) on $E$ by using the convolution with a fixed component. When $E$ is a Banach or Hilbert space, we add some hypotheses inspired in the fractal convolution of maps, and construct in this way convolved Schauder and Riesz bases, Bessel sequences and frames for the space.
\end{abstract}

Keywords: metric space; convolution; fractal; Schauder basis; Bessel sequence; Riesz basis; frames

MSC: 54E35; 46B15; 42C15; 28A80

\section{Introduction}

Fréchet [1] introduced the concept of metric space in 1906. A metric space is a set with specific structure determined by certain axioms. These axioms form an abstraction of the notion of distance, which empowers a unified treatment of diverse distinct cases under a single formalism. The nature of particular metric plays a crucial role in the investigation of various problems in differential geometry, non-Euclidean geometry, computer graphics, physics, mechanics, and engineering. The structure of metric space contains remarkable particular cases such as Banach and Hilbert spaces [2,3]. The latter are endowed with an additional structure of vector spaces that enriches the geometric structure, and their theory is the mathematical doctrine for non-relativistic quantum mechanics. Convolution is an important mathematical operation defined in various senses in the literature. Lahti [4] used the concept of discrete convolution in quasi-open sets to prove that every function of bounded variation (BV function) can be approximated in the $B V$ and $L^{\infty}$ norms by BV functions whose jump sets are of finite Hausdorff measure. Pap and Štajner [5] introduced the notion of generalized pseudo-convolution which can be used as a basic notion in many different theories of probabilistic metric spaces, information theory, fuzzy numbers, optimization, and system theory. In this paper, we consider binary operations in metric spaces satisfying properties as idempotency and inequalities related to the distance between operated elements with the same right or left factor (side inequalities). They are not related in any way with the topical addition and multiplication by a scalar. 
The association has been inspired by a fractal convolution defined in the framework of fractal interpolation functions (see the references [6,7]), generalizing it. The term "fractal convolution" is due to the curly forms typical of the graph of this type of mappings (See Figures 1 and 2), born from the cooperation of the functions implied. The proposed structure may find applications in the field of Discrete Mathematics, Function and Information Theory.

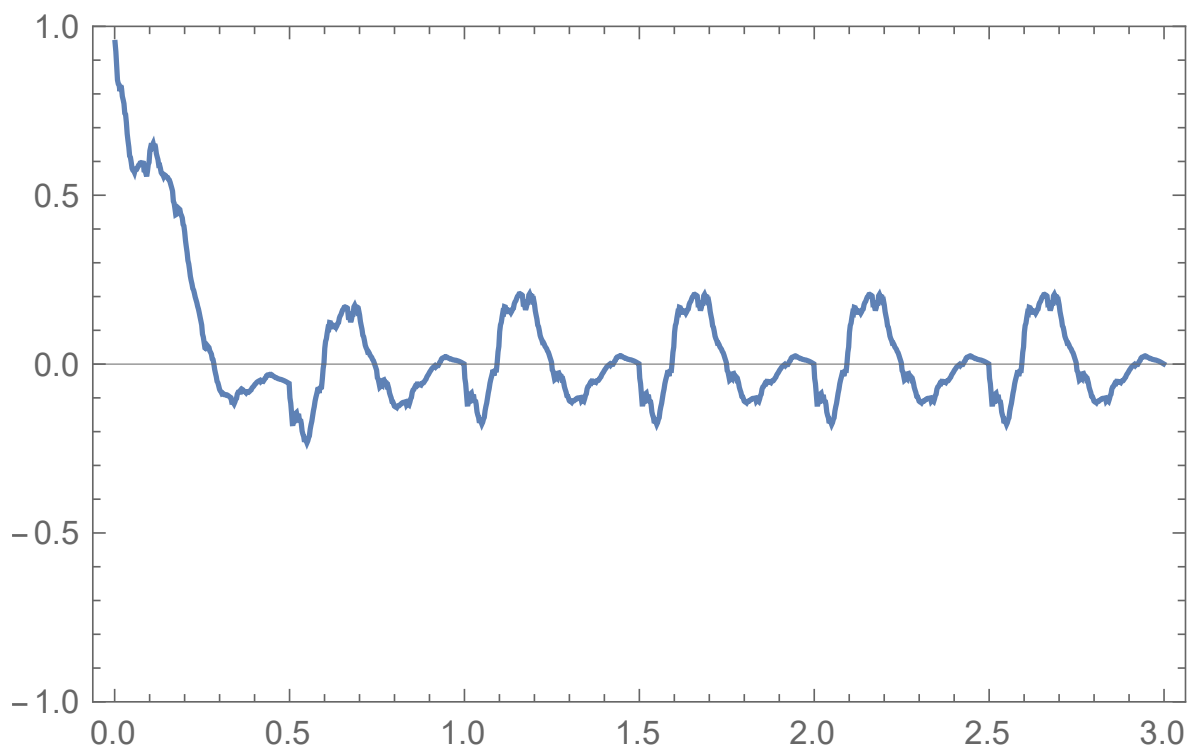

Figure 1. Fractal convolution $f \wedge b$ of the maps $f(t)=e^{-5 t} \cos \left(3 \pi \frac{t}{2}\right)$ and $b(t)=\cos \left(\pi \frac{t}{2}\right)$ in the interval $I=[0,3]$.

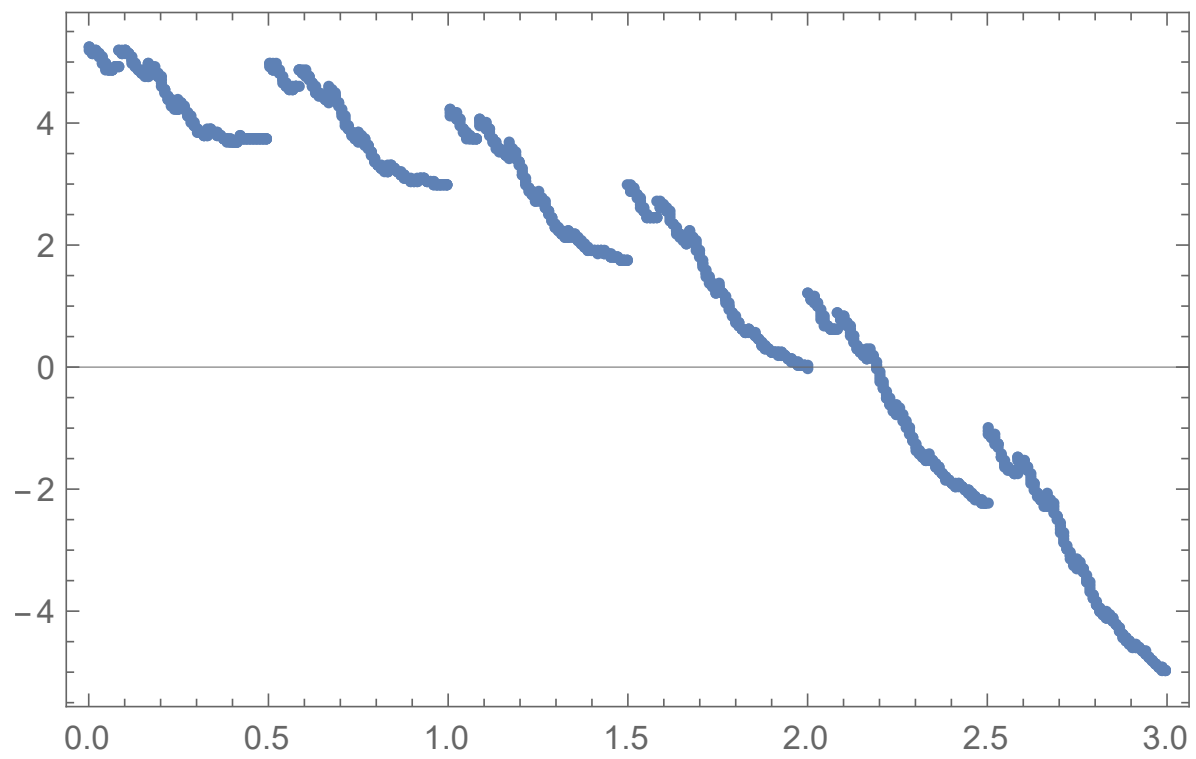

Figure 2. Fractal convolution $f \wedge b$ of the maps $f(t)=4-t^{2}$ and $b(t)=t^{2}-t-2$, in the interval $I=[0,3]$.

The convolution defined in this article does not enjoy, in general, the nice algebraic properties of the classical convolution of functions (associativity, commutativity, etc.). However it owns other properties that will be described in the following sections, in terms of the distance between operated and original elements. These properties are inherited by the convolution subsets. The classical convolution of two functions $f$ and $g$ computes basically a kind of weighted value of $f$ and in this sense may perform a smoothing on it. In the fractal convolution, $f$ represents a trend pattern and $g$ shapes the small oscillations around it (see Figures 1 and 2). Fractal convolution endows the graph of $f$ with a fractal 
structure, that enriches the geometric content of the same. Taking some "scale factors" of the model as zero, one obtains the component function $f$. In this sense, the fractal convolution provides a family of mappings that contains the original. This can be of great interest to approach optimization problems, due to the wider spectrum of the elements to be chosen.

There is an extensive bibliography about extensions of metric spaces (pseudo, quasi, generalized, ultra, fuzzy, probabilistic, etc.), however there are very few articles concerning operations in abstract metric spaces. Some authors generalize the concept of distance to maps of type $d: X \times X \rightarrow G$, where $G$ owns an algebraic structure composed of binary operations and/or relations ([8]). In the reference [9], the authors endow the interval $[0, \infty)$ with an operation, and this serves to define the concept of $T$-metric space. Triangular norms (t-norms) ([10]) are binary operations in $[0,1]$ that support the concept of fuzzy metric space, and opens a branch of the fuzzy logic. An extension is given in [11]. The reference [12] defines an $R$-metric related to an ordered algebraic structure.

As said previously, the references to operations in metric spaces are scarce. That is why this article may be of interest for the readers. Beginning from the concept of convolution, we define the self-map in the metric space $E$ by using the convolution with a fixed component (partial or one-sided convolution). Some fundamental properties of this type of operators are discussed in Section 2. Section 3 studies the convolution and partial convolution defined on subsets of $E$, showing some of their characteristics. For instance, we prove that a convolution can be induced on the space $\mathcal{H}(E)$ of non-empty compact subsets of $E$. In Section 4 , we assume that $E$ to be a normed vector space and the linearity in the operation. Then, the partial convolution induced by fixing the null element turns out to be a linear operator on $E$. The last part of this section is devoted to study the action of the above partial convolution on a Schauder basis. Finally, in Section 5, we assume $E$ to be a Hilbert space and we construct Bessel sequences and Riesz bases on $E$, and we prove that if $\left(e_{m}\right)$ is a frame, then the sequence of images by the partial operator applied on $\left(e_{m}\right)$ is also a frame (under certain conditions).

\section{Convolution in Metric Spaces}

Definition 1. Let $(E, d)$ be a metric space. A convolution is a binary operation $\wedge: E \times E \rightarrow E$, such that

1. $\wedge$ is idempotent: $e \wedge e=e, \forall e \in E$;

2. There exist $k, k^{\prime} \geq 0$, such that for any $e, e^{\prime}, e^{\prime \prime} \in E$,

$$
\begin{aligned}
& d\left(e \wedge e^{\prime}, e \wedge e^{\prime \prime}\right) \leq k d\left(e^{\prime}, e^{\prime \prime}\right), \\
& d\left(e^{\prime} \wedge e, e^{\prime \prime} \wedge e\right) \leq k^{\prime} d\left(e^{\prime}, e^{\prime \prime}\right) .
\end{aligned}
$$

\subsection{Particular Cases}

1. First we consider an example in Boolean Algebra, see for instance [13]. Let $Z_{2}$ (integers modulo 2) be the metric space with metric $d$ defined as follows: $d(0,0)=d(1,1)=0$, $d(0,1)=d(1,0)=c>0$. Let $\vee$ be the logical disjunction: $0 \vee 0=0,0 \vee 1=1,1 \vee 0=1$, $1 \vee 1=1$. This operator satisfies (1) and (2) with $k=k^{\prime}=1$,

$$
\begin{aligned}
& e=0, \begin{cases}e^{\prime}=0, e^{\prime \prime}=0 & \Rightarrow d\left(e \vee e^{\prime}, e \vee e^{\prime \prime}\right)=d(0,0)=0 \leq d\left(e^{\prime}, e^{\prime \prime}\right)=0 . \\
e^{\prime}=0, e^{\prime \prime}=1 & \Rightarrow d\left(e \vee e^{\prime}, e \vee e^{\prime \prime}\right)=d(0,1)=c \leq d\left(e^{\prime}, e^{\prime \prime}\right)=c . \\
e^{\prime}=1, e^{\prime \prime}=1 & \Rightarrow d\left(e \vee e^{\prime}, e \vee e^{\prime \prime}\right)=d(1,1)=0 \leq d\left(e^{\prime}, e^{\prime \prime}\right)=0 .\end{cases} \\
& e=1, \begin{cases}e^{\prime}=0, e^{\prime \prime}=0 & \Rightarrow d\left(e \vee e^{\prime}, e \vee e^{\prime \prime}\right)=d(1,1)=0 \leq d\left(e^{\prime}, e^{\prime \prime}\right)=0 . \\
e^{\prime}=0, e^{\prime \prime}=1 & \Rightarrow d\left(e \vee e^{\prime}, e \vee e^{\prime \prime}\right)=d(1,1)=0 \leq d\left(e^{\prime}, e^{\prime \prime}\right)=c . \\
e^{\prime}=1, e^{\prime \prime}=1 & \Rightarrow d\left(e \vee e^{\prime}, e \vee e^{\prime \prime}\right)=d(1,1)=0 \leq d\left(e^{\prime}, e^{\prime \prime}\right)=0 .\end{cases}
\end{aligned}
$$

In this case, $\vee$ is commutative. 
2. Let $\left(Z_{2}, d\right)$ be a metric space defined as in the above example and $\wedge$ be the logical conjunction on $E$ defined by $0 \wedge 0=0,0 \wedge 1=0,1 \wedge 0=0,1 \wedge 1=1$, then $\wedge$ is a commutative convolution operator on $E$;

3. Fractal convolution of functions: In many real-world phenomena, such as signal and image processing, economic and climatic series, bioelectric recordings, cartography, etc., the scientists need to manage irregular and sharp forms, and the standard functions often fall short to approach them. Barnsley [14] introduced the idea of data interpolation using the fractal methodology of iterated function systems. In this way, non-smooth self-similar functions were constructed in the last decades of the twentieth century. Nowadays fractal functions constitute a method of approximation of non-differentiable mappings, providing suitable tools for the description of irregular signals ([6,15-19]). Navascués [6] defined the fractal convolution of two maps defined on a real interval, on the basis of a special type of fractal interpolation function as follows:

Let $\Delta: t_{0}<t_{1} \cdots<t_{N}$ be any partition of the interval $I=\left[t_{0}, t_{N}\right]$ and let $I_{n}=\left[t_{n-1}, t_{n}\right]$, for all $n \in \mathbb{N}_{N}:=\{1,2, \ldots, N\}$. We define $L_{n}: I \rightarrow I_{n}$ by

$$
L_{n}(t)=a_{n} t+b_{n}
$$

where

$$
a_{n}=\frac{t_{n}-t_{n-1}}{t_{N}-t_{0}}, \quad b_{n}=\frac{t_{N} t_{n-1}-t_{0} t_{n}}{t_{N}-t_{0}} .
$$

Fix a scale function $\alpha \in\left(L^{\infty}(I)\right)^{N}$, we assume that

$$
C:=\operatorname{essup}\left\{\left|\alpha_{n}(t)\right|: t \in I ; n \in \mathbb{N}_{N}\right\}<1 \text {. }
$$

Now, let $L^{p}(I)$ be the Lebesgue space with the norm

$$
\|h\|_{p}:=\left(\int_{I}|h(t)|^{p} d t\right)^{1 / p} \text { for } 1 \leq p<\infty .
$$

For any $f, b \in L^{p}(I)$, define an operator $T_{f, b}: L^{p}(I) \rightarrow L^{p}(I)$ called Read-Bajraktarević (R-B) operator by

$$
T_{f, b} h(t):=f(t)+\left(\alpha_{n} \circ L_{n}^{-1}\right)(t) \cdot(h-b) \circ L_{n}^{-1}(t), \forall t \in I_{n}, n \in \mathbb{N}_{N} .
$$

Using the fact $C<1$, it can be verified that the R-B operator $T_{f, b}$ is a contractive map on the Banach space $\left(L^{p}(I),\|.\|_{p}\right)$. By the Banach contraction principle [20] (which states that every contraction map on a complete metric space has a unique fixed point), $T_{f, b}$ has a unique fixed point $f_{\Delta, b}^{\alpha} \in L^{p}(I)$.

For fixed $\Delta, \alpha$, define a binary operation $\wedge: L^{p}(I) \times L^{p}(I) \rightarrow L^{p}(I)$ by

$$
\wedge(f, b)=f \wedge b:=f_{\Delta, b}^{\alpha}
$$

The new map is called the fractal convolution of $f$ and $b$. It can be verified that the fractal convolution is a bounded linear operator (see the references $[6,7]$ ).

Proposition $1([7])$. Let $f, f^{\prime}, b, b^{\prime} \in L^{p}(I)$. Then

$$
\begin{aligned}
& \left\|f \wedge b-f \wedge b^{\prime}\right\|_{p} \leq \frac{C}{1-C}\left\|b-b^{\prime}\right\|_{p}, \\
& \left\|f \wedge b-f^{\prime} \wedge b\right\|_{p} \leq \frac{1}{1-C}\left\|f-f^{\prime}\right\|_{p} .
\end{aligned}
$$

That is $\wedge$ is a convolution on $L^{p}(I)$. 
Figure 1 represents the fractal convolution $f \wedge b$ of the functions $f(t)=e^{-5 t} \cos \left(3 \pi \frac{t}{2}\right)$ and $b(t)=\cos \left(\pi \frac{t}{2}\right)$ in the interval $I=[0,3]$. The partition $\Delta$ is uniform with $N=6$, and the scale functions are the maps $\alpha_{n}(t)=(3-t) / 10$ for all $n=1,2, \ldots, 6$. The result is a continuous function.

Figure 2 shows the fractal convolution $f \wedge b$ of the functions $f(t)=4-t^{2}$ and $b(t)=t^{2}-t-2$, in the interval $I=[0,3]$. The partition $\Delta$ is uniform with $N=6$, and the scale functions are the maps $\alpha_{n}(t)=(3-t) / 10$ for all $n=1,2, \ldots, 6$. The outcome is a discontinuous function.

4. $\quad$ Define a distance between two non-empty subsets $R$ and $S$ of a metric space $(E, d)$ by

$$
d(R, S):=\inf \{d(r, s): r \in R, s \in S\} .
$$

Note that $d$, defined as above, is not a metric. Let $C B(E)$ be the set of all non-empty closed and bounded subsets of $E$. Define $h: C B(E) \times C B(E) \rightarrow \mathbb{R}^{+}$, such that for any $R, S \in C B(E)$,

$$
h(R, S):=\max \left\{\sup _{r \in R} d(r, S), \sup _{s \in S} d(s, R)\right\} .
$$

Let $\mathcal{H}(E) \subset C B(E)$ be the set of all non-empty compact subsets of $E$. The map $h$ is a metric on $\mathcal{H}(E)$ (as well as on $C B(E)$ ) called the Hausdorff metric [21].

Lemma 1 ([22]). If $\left\{A_{i}\right\}_{i \in J}$ and $\left\{B_{i}\right\}_{i \in J}$ are finite collections of compact subsets of a metric space $(E, d)$, then $h\left(\cup_{i \in J} A_{i}, \cup_{i \in J} B_{i}\right) \leq \sup _{i \in J} h\left(A_{i}, B_{i}\right)$.

Define $\wedge: \mathcal{H}(E) \times \mathcal{H}(E) \rightarrow \mathcal{H}(E)$ by

$$
\wedge(R, S):=R \cup S, \quad \forall R, S \in \mathcal{H}(E) .
$$

Our claim is that $\wedge$ is a convolution on $\mathcal{H}(E)$ with respect to the Hausdorff metric. By using Lemma 1 , take $A_{1}=R, A_{2}=S, B_{1}=R$ and $B_{2}=S^{\prime}$, we obtain

$$
h\left(\wedge(R, S), \wedge\left(R, S^{\prime}\right)\right) \leq h\left(S, S^{\prime}\right) .
$$

Since $\wedge$ is commutative, we obtain

$$
h\left(\wedge(S, R), \wedge\left(S^{\prime}, R\right)\right) \leq h\left(S, S^{\prime}\right) .
$$

5. Let us consider an Euclidean space $E$. For two vectors $x, y \in E$ let us define their convolution as the element

$$
x \wedge y:=\frac{x+y}{2} .
$$

The inner operation so defined satisfies the conditions imposed to be a convolution, taking as $k$ and $k^{\prime}$ any numbers greater or equal than $1 / 2$.

\subsection{Some Properties of the Convolution Operation}

In this subsection, we study some elementary properties of the convolution and side convolutions in general metric spaces.

Note that, if $\wedge$ is a convolution on a metric space $(E, d)$ with constants $k, k^{\prime}$ as in Definition 1 , then for any $e, e^{\prime}, f, f^{\prime} \in E$,

$$
\begin{aligned}
& d\left(e \wedge e^{\prime}, f \wedge f^{\prime}\right) \leq d\left(e \wedge e^{\prime}, e \wedge f^{\prime}\right)+d\left(e \wedge f^{\prime}, f \wedge f^{\prime}\right) \\
\Rightarrow \quad & d\left(e \wedge e^{\prime}, f \wedge f^{\prime}\right) \leq k d\left(e^{\prime}, f^{\prime}\right)+k^{\prime} d(e, f) .
\end{aligned}
$$

We define a convolution operator $Q: E \times E \rightarrow E$ by

$$
Q\left(e, e^{\prime}\right)=e \wedge e^{\prime}, \quad \forall e, e^{\prime} \in E,
$$


and also define the partial convolution operators from $Q$ as follows:

Definition 2. Let $\wedge$ be a convolution on a metric space $(E, d)$, for any $e \in E$, define

$$
\begin{gathered}
Q_{e}^{1}\left(e^{\prime}\right):=Q\left(e, e^{\prime}\right): E \rightarrow E \\
e^{\prime} \rightarrow e \wedge e^{\prime},
\end{gathered}
$$

and

$$
\begin{gathered}
Q_{e}^{2}\left(e^{\prime}\right):=Q\left(e^{\prime}, e\right): E \rightarrow E \\
e^{\prime} \rightarrow e^{\prime} \wedge e .
\end{gathered}
$$

The operators $Q_{e}^{1}$ and $Q_{e}^{2}$ are called the partial or one-sided convolutions with $e$.

Hereafter, we will see some interesting properties of the above operators.

Proposition 2. If $(E, d)$ is a metric space and $Q$ is a convolution operator on $E \times E$ as defined in the above, then the operator $Q$ is a Lipschitz function with Lipschitz constant $\left(k+k^{\prime}\right)$ with respect to the Euclidean metric $d_{2}$. In particular, $Q$ is continuous.

Proof. Let $e, e^{\prime}, f, f^{\prime} \in E$. By (3), we have

$$
\begin{aligned}
d\left(e \wedge e^{\prime}, f \wedge f^{\prime}\right) & \leq k d\left(e^{\prime}, f^{\prime}\right)+k^{\prime} d(e, f) \\
& \leq k \sqrt{[d(e, f)]^{2}+\left[d\left(e^{\prime}, f^{\prime}\right)\right]^{2}}+k^{\prime} \sqrt{[d(e, f)]^{2}+\left[d\left(e^{\prime}, f^{\prime}\right)\right]^{2}} \\
& =\left(k+k^{\prime}\right) d_{2}\left(\left(e, e^{\prime}\right),\left(f, f^{\prime}\right)\right) .
\end{aligned}
$$

Proposition 3. For any $e \in E$, the operators $Q_{e}^{1}$ and $Q_{e}^{2}$ are Lipschitz functions with Lipschitz constants $k$ and $k^{\prime}$, respectively. In particular $Q_{e}^{1}$ and $Q_{e}^{2}$ are contractive if $k<1$ and $k^{\prime}<1$, respectively.

Proof. Let $e, e^{\prime} \in E$, by the definition of convolution,

$$
\begin{aligned}
d\left(Q_{e}^{1}\left(e^{\prime}\right), Q_{e}^{1}\left(e^{\prime \prime}\right)\right) & =d\left(e \wedge e^{\prime}, e \wedge e^{\prime \prime}\right) \\
& \leq k d\left(e^{\prime}, e^{\prime \prime}\right) .
\end{aligned}
$$

Similarly, $d\left(Q_{e}^{2}\left(e^{\prime}\right), Q_{e}^{2}\left(e^{\prime \prime}\right)\right)=d\left(e^{\prime} \wedge e, e^{\prime \prime} \wedge e\right) \leq k^{\prime} d\left(e^{\prime}, e^{\prime \prime}\right)$.

Consequently, the convolution and the partial convolutions are uniformly continuous maps.

\section{Convolution of Sets}

Now we define convolution on sets as in the following way and discuss its properties. Take any $R, S \subset E$ and define

$$
\begin{gathered}
R \wedge e:=\{r \wedge e: r \in R\}, \\
e \wedge S:=\{e \wedge s: s \in S\}, \\
R \wedge S:=\{r \wedge s: r \in R, s \in S\} .
\end{gathered}
$$

The following are basic properties of the convolution sets:

1. Since $e \wedge e=e$, if $e \in R$, then $e \in R \wedge e$;

2. If $e \in S$, then $e \in e \wedge S$; 
3. Consequently, for any subsets $R, S \subset E$,

$$
(R \cap S) \subseteq(R \wedge S) \cap(S \wedge R)
$$

Proposition 4. If $R, S$ are subsets of a metric space $(E, d)$, then for any $e, e^{\prime}$ :

$$
\begin{aligned}
& d\left(R \wedge e, R \wedge e^{\prime}\right) \leq k d\left(e, e^{\prime}\right), \\
& d\left(e \wedge S, e^{\prime} \wedge S\right) \leq k^{\prime} d\left(e, e^{\prime}\right) .
\end{aligned}
$$

Proof. First, we prove the first inequality:

$$
d\left(R \wedge e, R \wedge e^{\prime}\right)=\inf _{r, r^{\prime} \in R} d\left(r \wedge e, r^{\prime} \wedge e^{\prime}\right) \leq \inf _{r \in R} d\left(r \wedge e, r \wedge e^{\prime}\right) \leq k d\left(e, e^{\prime}\right) .
$$

The second inequality can be proved similarly.

Proposition 5. Let $R, S$ be subsets of $E$. Then, for any $e \in E$ :

$$
\begin{aligned}
& d(R, R \wedge e) \leq k d(R, e), \\
& d(S, e \wedge S) \leq k^{\prime} d(S, e) .
\end{aligned}
$$

Proof. For the first result, consider

$$
d(R, R \wedge e)=\inf _{r, r^{\prime} \in R} d\left(r, r^{\prime} \wedge e\right) \leq \inf _{r \in R} d(r \wedge r, r \wedge e) \leq \inf _{r \in R} k d(r, e)=k d(R, e) .
$$

Similarly, the second inequality can be proved.

By Propositions 2 and 3, for any $e \in E, Q, Q_{e}^{1}$, and $Q_{e}^{2}$ are continuous. From that, suppose $R, S$ are compact subsets of $E$, then $Q(R \times S)=R \wedge S, Q_{e}^{1}(S)=e \wedge S$ and $Q_{e}^{2}(R)=$ $R \wedge e$ are also compact sets.

Proposition 6. Suppose $R, R^{\prime}, S, S^{\prime} \in \mathcal{H}(E)$, then for any e, $e^{\prime} \in E$, the Hausdorff metric satisfies the following properties with respect to the convolution:

1. $h\left(R, R \wedge e^{\prime}\right) \leq k h\left(R, e^{\prime}\right)$,

2. $h(S, e \wedge S) \leq k^{\prime} h(S, e)$,

3. $h(R, R \wedge S) \leq k h(R, S)$,

4. $h(S, R \wedge S) \leq k^{\prime} h(R, S)$,

5. $h\left(R \wedge S, R \wedge S^{\prime}\right) \leq k h\left(S, S^{\prime}\right)$,

6. $h\left(R \wedge S, R^{\prime} \wedge S\right) \leq k^{\prime} h\left(R, R^{\prime}\right)$,

7. $h\left(R \wedge S, R^{\prime} \wedge S^{\prime}\right) \leq k^{\prime} h\left(R, R^{\prime}\right)+k h\left(S, S^{\prime}\right)$.

Proof. Consider the last inequality, let $r \in R, s \in S$, then

$$
\begin{aligned}
d\left(r \wedge s, R^{\prime} \wedge S^{\prime}\right) & =\inf _{r^{\prime} \in R^{\prime}, s^{\prime} \in S^{\prime}} d\left(r \wedge s, r^{\prime} \wedge s^{\prime}\right) \\
& \leq \inf _{r^{\prime} \in R^{\prime}, s^{\prime} \in S^{\prime}}\left(d\left(r \wedge s, r^{\prime} \wedge s\right)+d\left(r^{\prime} \wedge s, r^{\prime} \wedge s^{\prime}\right)\right) \\
& \leq k^{\prime} d\left(r, R^{\prime}\right)+k d\left(s, S^{\prime}\right) \\
& \leq k^{\prime} h\left(R, R^{\prime}\right)+k h\left(S, S^{\prime}\right),
\end{aligned}
$$

and let $r^{\prime} \in R^{\prime}, s^{\prime} \in S^{\prime}$, we have

$$
\begin{aligned}
d\left(r^{\prime} \wedge s^{\prime}, R \wedge S\right) & =\inf _{r \in R, s \in S} d\left(r^{\prime} \wedge s^{\prime}, r \wedge s\right) \leq k^{\prime} d\left(r^{\prime}, R\right)+k d\left(s^{\prime}, S\right) \\
& \leq k^{\prime} h\left(R, R^{\prime}\right)+k h\left(S, S^{\prime}\right) .
\end{aligned}
$$


Hence, the last inequality is proved. The other items are particular cases of the last inequality.

Theorem 1. If $\wedge$ is a convolution on a metric space $(E, d)$, then $\wedge$ is also a convolution on the Hausdorff metric space $(\mathcal{H}(E), h)$ with the same constants $k, k^{\prime}$.

Example 1. Let us consider a finite (compact) system of polynomials in $\mathcal{L}^{p}(I), R=\left(p_{n}\right)_{n=0}^{N}$ ' and a family of modified "polynomials" $\widetilde{R}=\left\{p_{n} \wedge t_{m}: n=0, \ldots N ; m=0, \ldots M\right\}$, where $S=\left(t_{m}\right)_{n=0}^{M}$ is a compact set of trigonometric functions. The fractal convolution with $t_{m}$ will provide additional frequencies to the elements of $R$, in a process similar to the convolution depicted in Figure 1. The Hausdorff distance between original and modified systems $R$ and $\widetilde{R}$ will be bounded by the distance between $R$ and $S$ multiplied by $k=C /(1-C)$ (see item 3 of Section 2.1), according to the third item of the previous proposition.

The convolution operator $\wedge$ can be substituted by the union in Properties $1-7$ of Proposition 6 according to Section 2.1, Item 4 by taking $k=k^{\prime}=1$. In this case, $R$ and $S$ would be sets composed of compact sets.

\section{Convolution in Normed Spaces}

Now, let us consider that $(E,\|\|$.$) is a normed linear space and assume that the convo-$ lution operator

$$
\begin{aligned}
& Q: E \times E \rightarrow E, \\
& \left(e, e^{\prime}\right) \rightarrow e \wedge e^{\prime},
\end{aligned}
$$

is linear. Define partial convolutions with null element 0 ,

$$
Q_{0}^{1}: E \rightarrow E \text { as } Q_{0}^{1}(e):=Q(0, e)=0 \wedge e,
$$

and

$$
Q_{0}^{2}: E \rightarrow E \text { as } Q_{0}^{2}(e):=Q(e, 0)=e \wedge 0 .
$$

The linearity of the convolution operator $Q$ implies that $Q_{0}^{1}$ and $Q_{0}^{2}$ are also linear operators. Now, we check the boundedness of $Q, Q_{0}^{1}$ and $Q_{0}^{2}$ (though we know they are continuous).

First, consider the convolution operator $Q$, by (3),

$$
\begin{aligned}
\left\|Q\left(e, e^{\prime}\right)\right\| & =\left\|e \wedge e^{\prime}-0 \wedge 0\right\| \leq k\left\|e^{\prime}-0\right\|+k^{\prime}\|e-0\| \\
& \leq\left(k+k^{\prime}\right)\left\|\left(e, e^{\prime}\right)-(0,0)\right\|_{p}=\left(k+k^{\prime}\right)\left\|\left(e, e^{\prime}\right)\right\|_{p},
\end{aligned}
$$

where

$$
\begin{gathered}
\left\|(e, s)-\left(e^{\prime}, s^{\prime}\right)\right\|_{p}:=\left(\left\|e-e^{\prime}\right\|^{p}+\left\|s-s^{\prime}\right\|^{p}\right)^{1 / p}, \text { if } 1 \leq p<\infty, \\
\left\|(e, s)-\left(e^{\prime}, s^{\prime}\right)\right\|_{p}:=\max \left\{\left\|e-e^{\prime}\right\|,\left\|s-s^{\prime}\right\|\right\}, \text { if } \quad p=\infty .
\end{gathered}
$$

This implies $Q$ is bounded with respect to $\|\cdot\|_{p}$ and the norm is bounded by $k+k^{\prime}$, that is

$$
\|Q\| \leq k+k^{\prime}
$$

Similarly, for the boundedness of partial convolutions $Q_{0}^{1}$ and $Q_{0}^{2}$, we have

$$
\left\|Q_{0}^{1}(e)\right\|=\|0 \wedge e-0 \wedge 0\| \leq k\|e\|,
$$

and

$$
\left\|Q_{0}^{2}(e)\right\|=\|e \wedge 0-0 \wedge 0\| \leq k^{\prime}\|e\| .
$$

This implies that the norms of $Q_{0}^{1}$ and $Q_{0}^{2}$ are bounded by $k, k^{\prime}$, respectively, that is

$$
\left\|Q_{0}^{1}\right\| \leq k \text { and }\left\|Q_{0}^{2}\right\| \leq k^{\prime} .
$$


As said before, $Q_{0}^{1}$ and $Q_{0}^{2}$ are contractive if $k<1$ and $k^{\prime}<1$, respectively, and $Q$ is contractive if $k+k^{\prime}<1$.

Since $Q$ is linear, for all $e \in E$

$$
Q_{0}^{1}(e)+Q_{0}^{2}(e)=Q(0, e)+Q(e, 0)=Q(e, e)=e .
$$

Therefore,

$$
Q_{0}^{1}+Q_{0}^{2}=I
$$

Proposition 7. If $k^{\prime}<1$, then $Q_{0}^{1}$ is a topological isomorphism. If $k<1$, then $Q_{0}^{2}$ is also.

Proof. It is a straightforward consequence of the previous results.

\subsection{Other Properties of $Q_{0}^{1}$ and $Q_{0}^{2}$}

In this subsection, we consider a convolution satisfying an additional condition: There exists $c \in \mathbb{R}$ such that for any $e \in E$,

$$
c\|e \wedge 0\|=\|0 \wedge e\| .
$$

This condition is inspired by the properties of the fractal convolution of functions. The inequality (5) implies that

$$
\left\|Q_{0}^{1}\right\|=c\left\|Q_{0}^{2}\right\| .
$$

All bounds (upper and lower) of $\left\|Q_{0}^{1}\right\|$ are applicable to $\left\|Q_{0}^{2}\right\|$ multiplied by $c$. As said before, we assume $Q$ is linear in this section, that is to say,

$$
(e+f) \wedge\left(e^{\prime}+f^{\prime}\right)=\left(e \wedge e^{\prime}\right)+\left(f \wedge f^{\prime}\right) .
$$

In particular,

$$
\begin{aligned}
& e-0 \wedge e=e \wedge e-0 \wedge e=e \wedge 0, \\
& e-e \wedge 0=e \wedge e-e \wedge 0=0 \wedge e .
\end{aligned}
$$

By (5)-(7), we have

$$
\begin{aligned}
& c\|0 \wedge e-e\|=\|0 \wedge e\|, \\
& \|e \wedge 0-e\|=c\|e \wedge 0\| .
\end{aligned}
$$

By (8), we obtain

$$
\left\|Q_{0}^{1}(e)\right\| \leq c\left\|Q_{0}^{1}(e)\right\|+c\|e\|,
$$

this implies

$$
\left\|Q_{0}^{1}(e)\right\| \leq \frac{c}{1-c}\|e\|,
$$

and

$$
\left\|Q_{0}^{1}\right\| \leq \frac{c}{1-c}
$$

if $c<1$. Since $I=Q_{0}^{1}+Q_{0}^{2}$

$$
1 \leq(1+1 / c)\left\|Q_{0}^{1}\right\| \Rightarrow \frac{c}{1+c} \leq\left\|Q_{0}^{1}\right\| .
$$

Similarly, by (9), we have

$$
\left\|Q_{0}^{2}(e)\right\| \leq \frac{1}{1-c}\|e\|,
$$

and, thus,

$$
\left\|Q_{0}^{2}\right\| \leq \frac{1}{1-c}
$$


if $c<1$ and

$$
\frac{1}{1+c} \leq\left\|Q_{0}^{2}\right\|
$$

We know also that $\left\|Q_{0}^{1}\right\| \leq k$ and $\left\|Q_{0}^{2}\right\| \leq k^{\prime}$, therefore, if $c<1$, we have

$$
\begin{aligned}
& \frac{c}{1+c} \leq\left\|Q_{0}^{1}\right\| \leq \min \left\{k, \frac{c}{1-c}\right\} . \\
& \frac{1}{1+c} \leq\left\|Q_{0}^{2}\right\| \leq \min \left\{k^{\prime}, \frac{1}{1-c}\right\} .
\end{aligned}
$$

According to (10) if $c<1 / 2, Q_{0}^{2}$ is a topological isomorphism.

Proposition 8. If $c \neq 0$, then $Q_{0}^{1}$ is injective.

Proof. Suppose $Q_{0}^{1}(e)=0$, this implies by (8) that $c\|0 \wedge e-e\|=\|0 \wedge e\|=0$. Since $c \neq 0$, $0 \wedge e=e$, and $Q_{0}^{1}(e)=0 \wedge e=0$, then $e=0$.

Proposition 9. If $E$ is Banach, then $Q_{0}^{1}$ has a closed range.

Proof. Suppose $c=0$, then $Q_{0}^{1}$ is the null-operator. Assume that $c \neq 0$, by (8)

$$
c(\|e\|-\|0 \wedge e\|) \leq\|0 \wedge e\|,
$$

and, therefore,

$$
\|e\| \leq \frac{1+c}{c}\left\|Q_{0}^{1}(e)\right\| .
$$

Let $\left(Q_{0}^{1}\left(e_{m}\right)\right)$ converges to $e^{\prime}$. By linearity of $Q_{0}^{1}$

$$
\left\|e_{m}-e_{n}\right\| \leq \frac{1+c}{c}\left\|Q_{0}^{1}\left(e_{m}\right)-Q_{0}^{1}\left(e_{n}\right)\right\| .
$$

This gives $\left(e_{m}\right)$ is a Cauchy sequence and hence it converges to some point $e$ in $E$. Using the continuity of $Q_{0}^{1}$, we conclude $e^{\prime}=Q_{0}^{1}(e)$.

Proposition 10. If $E$ is Banach, then $Q_{0}^{2}$ is injective and has a closed range.

Proof. Assume $e \wedge 0=0$, this implies by (9) that $\|e \wedge 0-e\|=c\|e \wedge 0\|=0$ and therefore $e=0$. Hence, the operator $Q_{0}^{2}$ is injective. Additionally,

$$
\|e\|-\|e \wedge 0\| \leq c\|e \wedge 0\|,
$$

and

$$
\|e\| \leq(1+c)\left\|Q_{0}^{2}(e)\right\| .
$$

From the above inequality and the proof of Proposition 9, we conclude $Q_{0}^{2}$ has a closed range.

Proposition 11 ([23]). Let $T$ be a linear operator on a Banach space E. Suppose there exist $c_{1}, c_{2} \in[0,1)$, such that

$$
\|T e-e\| \leq c_{1}\|e\|+c_{2}\|T e\|, \quad \forall e \in E .
$$

Then, $T$ is topological isomorphism, and for all $e \in E$,

$$
\begin{gathered}
\frac{1-c_{1}}{1+c_{2}}\|e\| \leq\|T e\| \leq \frac{1+c_{1}}{1-c_{2}}\|e\|, \\
\frac{1-c_{2}}{1+c_{1}}\|e\| \leq\left\|T^{-1} e\right\| \leq \frac{1+c_{2}}{1-c_{1}}\|e\| .
\end{gathered}
$$


Theorem 2. Let $E$ be a Banach space. If $c>1$, then $Q_{0}^{1}$ is a topological isomorphism, and it satisfies the following inequality for all $e \in E$ :

$$
\begin{gathered}
\frac{c}{c+1}\|e\| \leq\left\|Q_{0}^{1}(e)\right\| \leq \frac{c}{c-1}\|e\|, \\
\frac{c-1}{c}\|e\| \leq\left\|\left(Q_{0}^{1}\right)^{-1}(e)\right\| \leq \frac{c+1}{c}\|e\| .
\end{gathered}
$$

If $c<1$, then $Q_{0}^{2}$ is a topological isomorphism, and it satisfies the following inequality for all $e \in E$ :

$$
\begin{gathered}
\frac{1}{1+c}\|e\| \leq\left\|Q_{0}^{2}(e)\right\| \leq \frac{1}{1-c}\|e\|, \\
(1-c)\|e\| \leq\left\|\left(Q_{0}^{2}\right)^{-1}(e)\right\| \leq(1+c)\|e\| .
\end{gathered}
$$

Proof. From (8) and (9), we have $c_{1}=0, c_{2}=1 / c$ and $c_{1}=0, c_{2}=c$ in Proposition 11 for the linear operator $Q_{0}^{1}$ and $Q_{0}^{2}$, respectively.

Consequence: If $c>1,\left\|Q_{0}^{1}\right\| \leq \frac{c}{c-1}$.

Example 2. According to Lemma 6.3 of the reference [24], if $\left|\alpha_{n}(t)\right|=C$, for all $n$ and $t$, the fractal convolution satisfies the equality (5) for $c=C$ since $\|0 \wedge f\|=C\|0 \wedge f-f\|=C\|f \wedge 0\|$, for any $f \in \mathcal{L}^{2}([-1,1])$. Since $C<1$ (see item 3 of Section 2.1) the right convolution with the null function in $\mathcal{L}^{2}([-1,1])$ is a topological isomorphism. If $\left(p_{m}\right)$ is the Legendre basis of polynomials, then $\left(p_{m} \wedge f_{0}\right)$ is also a basis for the space $\mathcal{L}^{2}([-1,1])$, where $f_{0}$ represents the null function.

\subsection{Schauder Bases of Convolution}

Definition 3. A sequence $\left(e_{m}\right)_{m=1}^{\infty}$ in a normed space $E$ is called a Schauder basis for $E$ if for each $e$ in $E$, there is a unique sequence $\left(c_{m}\right)$ of scalars, such that $e=\sum_{m=1}^{\infty} c_{m} e_{m}$.

Definition 4. A subset $S$ of a vector space $E^{\prime}$ over a field $F$ is called a spanning family of $E$ if

$$
E^{\prime}=\left\{\sum_{m=1}^{n} c_{m} e_{m}: n \in \mathbb{N}, c_{m} \in F, e_{m} \in S\right\} .
$$

In such case, we say $E^{\prime}$ is a span of $S$.

Definition 5. A sequence $\left(e_{m}\right)$ in a normed space E is called a Schauder sequence if it is a Schauder basis for the closed span of $\left(e_{m}\right)$.

Proposition 12. If $c \neq 0$, and $\left(e_{m}\right)$ is a Schauder basis of $E$, then $\left(0 \wedge e_{m}\right)$ is a Schauder sequence of $E$.

Proof. By Propositions 8 and 9, we conclude that $Q_{0}^{1}$ is a topological isomorphism on its range. Hence, $\left(Q_{0}^{1}\left(e_{m}\right)\right)$ is a Schauder basis for the range of $Q_{0}^{1}$.

Proposition 13. If $c<1$ or $k<1$ and $\left(e_{m}\right)$ is a (bounded) Schauder basis of E Banach, then $\left(e_{m} \wedge 0\right)=\left(e_{m}-0 \wedge e_{m}\right)$ is also a (bounded) Schauder basis of $E$.

Proof. By (4), $Q_{0}^{2}\left(e_{m}\right)=\left(e_{m}-Q_{0}^{1}\left(e_{m}\right)\right)$. If $c<1, Q_{0}^{2}$ is a topological isomorphism due to Theorem 2. This implies that $\left(e_{m}-0 \wedge e_{m}\right)$ is a Schauder basis of $E$.

Assume $\left(e_{m}\right)$ is bounded, then there exist $M_{l}$ and $M_{u}$, such that

$$
M_{l} \leq\left\|e_{m}\right\| \leq M_{u}
$$


From the definition of convolution, we obtain

$$
\left\|e_{m}-0 \wedge e_{m}\right\| \leq k^{\prime}\left\|e_{m}\right\| \leq k^{\prime} M_{u}
$$

Consider

$$
\begin{aligned}
\left\|e_{m}-0 \wedge e_{m}\right\| & \geq\left\|e_{m}\right\|-\left\|0 \wedge e_{m}\right\| \\
& =\left\|e_{m}\right\|-c\left\|0 \wedge e_{m}-e_{m}\right\|,
\end{aligned}
$$

and

$$
(1+c)\left\|e_{m}-0 \wedge e_{m}\right\| \geq\left\|e_{m}\right\| \geq M_{l} .
$$

If $k<1, Q_{0}^{2}$ is also an isomorphism by Proposition 7 , and we obtain the same result.

In general, in all the cases where $Q_{0}^{1}$ or $Q_{0}^{2}$ are isomorphisms, the bases are preserved by the respective side convolution with zero.

\section{Convolution in Hilbert Spaces}

Consider now the case where $E$ is a separable Hilbert space and we will discuss the properties of the operators $Q_{0}^{1}$ and $Q_{0}^{2}$. In this section, we discuss the existence of convolved bases, Bessel sequences, frames, etc. For these definitions we refer to $([25,26])$. As in Section 4, we consider that the operator $Q$ is linear and satisfying the condition (5), with subsequent identities (8) and (9).

Definition 6. A sequence $\left(e_{m}\right)$ is called a Bessel sequence in a Hilbert space E if there exists a constant $B>0$ such that for all $e \in E$,

$$
\left.\sum_{m=1}^{\infty}\left|<e, e_{m}\right\rangle\right|^{2} \leq B\|e\|^{2}
$$

Definition 7. A sequence $\left(e_{m}\right)$ is called a Riesz basis in a Hilbert space $E$ if it is equivalent to an orthonormal basis $\left(r_{m}\right)$ of $E$, that is to say, there exists an operator $T$ linear, bijective and bicontinuous (topological isomorphism), such that $T\left(e_{m}\right)=r_{m}$.

Definition 8. A sequence $\left(e_{m}\right)$ in a Hilbert space $E$, is called a Riesz sequence if there exist $k_{1}, k_{2}>0$, such that for any $\left(c_{m}\right) \in l^{2}$,

$$
k_{1} \sum_{m=1}^{\infty}\left|c_{m}\right|^{2} \leq\left\|\sum_{m=1}^{\infty} c_{m} e_{m}\right\|^{2} \leq k_{2} \sum_{m=1}^{\infty}\left|c_{m}\right|^{2} .
$$

Proposition 14. If $\left(e_{m}\right)$ is an orthonormal basis of $E$, then $\left(0 \wedge e_{m}\right)$ is a Riesz sequence in $E$.

Proof. Let $e=\sum_{m=1}^{\infty} c_{m} e_{m}$ in $E$ for any $\left(c_{m}\right) \in l^{2}$. Since the operator

$$
Q_{1}^{0}(e)=\sum_{m=1}^{\infty} c_{m}\left(0 \wedge e_{m}\right)
$$

is bounded and $\left(e_{m}\right)$ is an orthonormal basis,

$$
\left\|\sum_{m=1}^{\infty} c_{m}\left(0 \wedge e_{m}\right)\right\|^{2} \leq\left\|Q_{0}^{1}\right\|^{2}\|e\|^{2}=\left\|Q_{0}^{1}\right\|^{2} \sum_{m=1}^{\infty}\left|c_{m}\right|^{2} .
$$

From (8), we have

$$
c(\|e\|-\|0 \wedge e\|) \leq\|0 \wedge e\|,
$$

this implies

$$
\frac{c}{1+c}\|e\| \leq\|0 \wedge e\| .
$$


Hence,

$$
k_{1} \sum_{m=1}^{\infty}\left|c_{m}\right|^{2} \leq\left\|\sum_{m=1}^{\infty} c_{m}\left(0 \wedge e_{m}\right)\right\|^{2} \leq k_{2} \sum_{m=1}^{\infty}\left|c_{m}\right|^{2},
$$

where $k_{1}=\left(\frac{c}{1+c}\right)^{2}$ and $k_{2}=\left\|Q_{0}^{1}\right\|^{2} \leq k^{2}$.

Example 3. Haar system is an orthonormal basis of $\mathcal{L}^{2}([0,1])$ composed of discontinuous functions ([27]). Then the set of convolved elements $\left(f_{0} \wedge H_{m}\right)$, where $f_{0}$ is the null function, is a Riesz sequence of the same space.

Proposition 15. If $\left(e_{m}\right)$ is an orthonormal basis of $E$, then $\left(e_{m} \wedge 0\right)$ is a Bessel sequence in $E$.

Proof. Let $e \in E$. Since $\left(e_{m}\right)$ is an orthonormal basis, we have

$$
\begin{aligned}
\sum_{m=1}^{\infty}\left|<e, Q_{0}^{2}\left(e_{m}\right)>\right|^{2} & =\sum_{m=1}^{\infty}\left|<\left(Q_{0}^{2}\right)^{*}(e), e_{m}>\right|^{2} \\
& \leq\left\|\left(Q_{0}^{2}\right)^{*}(e)\right\|^{2} \\
& \leq\left\|\left(Q_{0}^{2}\right)^{*}\right\|^{2}\|e\|^{2}
\end{aligned}
$$

The Bessel constant is $\left\|\left(Q_{0}^{2}\right)^{*}\right\|^{2}=\left\|\left(Q_{0}^{2}\right)\right\|^{2}$, where $\left(Q_{0}^{2}\right)^{*}$ is the adjoint operator of $Q_{0}^{2}$.

Example 4. According to this proposition, the sequence of convolutions $\left(H_{m} \wedge f_{0}\right)$, where $f_{0}$ is the null function, is a Bessel sequence of $\mathcal{L}^{2}([0,1])$.

Theorem 3. If $\left(e_{m}\right)$ is a Riesz sequence in $E$, then $\left(e_{m} \wedge 0\right)$ is also a Riesz sequence.

Proof. We know that $Q_{0}^{2}$ is injective, and it has a closed range (cf. Proposition 10). Therefore, it is a topological isomorphism from $\left[e_{m}\right]$ onto $\left[Q_{0}^{2}\left(e_{m}\right)\right]$ and hence it preserves bases.

Two sequences $\left(e_{m}\right)$ and $\left(r_{m}\right)$ are said to be biorthogonal in a Hilbert space $E$ if

$$
<e_{m}, r_{k}>=\delta_{m, k}:= \begin{cases}1 & \text { if } m=k, \\ 0 & \text { if } m \neq k\end{cases}
$$

Theorem 4 ([25]). Assume that $\left(e_{m}\right)$ is a basis for E. Then, there exists a unique family $\left(r_{m}\right)$ in $E$, such that

$$
e=\sum_{m=1}^{\infty}<e, r_{m}>e_{m}, \forall e \in E .
$$

$\left(r_{m}\right)$ is a basis for $E$, and $\left(e_{m}\right)$ and $\left(r_{m}\right)$ are biorthogonal.

Proposition 16. If $c<1$ or $k<1$ and $\left(e_{m}\right)$ is a basis of $E$, then there exists a unique family $\left(r_{m}\right)$ in $E$, such that

$$
e=\sum_{m=1}^{\infty}<e,\left(\left(Q_{0}^{2}\right)^{-1}\right)^{*}\left(r_{m}\right)>\left(e_{m} \wedge 0\right), \forall e \in E
$$

$\left(\left(\left(Q_{0}^{2}\right)^{-1}\right)^{*}\left(r_{m}\right)\right)$ is a basis for $E$ and $\left(e_{m} \wedge 0\right)$ and $\left(\left(\left(Q_{0}^{2}\right)^{-1}\right)^{*}\left(r_{m}\right)\right)$ are biorthogonal.

Proof. Let $e \in E$. Since $Q_{0}^{2}$ is a topological isomorphism (see Theorem 2 and Proposition 7), take $s=\left(Q_{0}^{2}\right)^{-1}(e)$. By Theorem 4, there exists a unique family $\left(r_{m}\right)$ in $E$, being a basis, such that

$$
r=\sum_{m=1}^{\infty}<r, r_{m}>e_{m} \forall r \in E .
$$


Therefore,

$$
\begin{aligned}
e=Q_{0}^{2}(s) & =\sum_{m=1}^{\infty}<s, r_{m}>\left(e_{m} \wedge 0\right) \\
& =\sum_{m=1}^{\infty}<\left(Q_{0}^{2}\right)^{-1}(e), r_{m}>\left(e_{m} \wedge 0\right) \\
& =\sum_{m=1}^{\infty}<e,\left(\left(Q_{0}^{2}\right)^{-1}\right)^{*}\left(r_{m}\right)>\left(e_{m} \wedge 0\right) .
\end{aligned}
$$

Since $\left(r_{m}\right)$ is a basis for $E,\left(\left(\left(Q_{0}^{2}\right)^{-1}\right)^{*}\left(r_{m}\right)\right)$ is a basis for E. Applying Theorem 4, to the basis $\left(e_{m} \wedge 0\right)$ and from (15), we obtain $\left(\left(\left(Q_{0}^{2}\right)^{-1}\right)^{*}\left(r_{m}\right)\right)$ is unique, and $\left(e_{m} \wedge 0\right)$, $\left(\left(\left(Q_{0}^{2}\right)^{-1}\right)^{*}\left(r_{m}\right)\right)$ are biorthogonal.

Frames and convolution: Here we study some relations between frames and convolution.

Definition 9. A sequence $\left(e_{m}\right)$ in a separable Hilbert space $E$ is called a frame if there exists frame bounds $A, B \in \mathbb{R}^{+}$such that for any $e \in E$,

$$
A\|e\|^{2} \leq\left.\sum_{m=1}^{\infty}\left|<e, e_{m}\right\rangle\right|^{2} \leq B\|e\|^{2} .
$$

Proposition 17. If $c>1$ or $k^{\prime}<1$ and $\left(e_{m}\right)$ is a frame for $E$ with frame bounds $A, B$, then $\left(0 \wedge e_{m}\right)$ is a frame for $E$ with frame bounds $A\left\|\left(Q_{0}^{1}\right)^{-1}\right\|^{-2}, B\left\|Q_{0}^{1}\right\|^{2}$.

Proof. Let $e \in E$. Now

$$
\begin{aligned}
\sum_{m=1}^{\infty}\left|<e, Q_{0}^{1}\left(e_{m}\right)>\right|^{2} & =\sum_{m=1}^{\infty}\left|<\left(Q_{0}^{1}\right)^{*}(e), e_{m}>\right|^{2} \\
& \leq B\left\|\left(Q_{0}^{1}\right)^{*}(e)\right\|^{2} \\
& \leq B\left\|Q_{0}^{1}\right\|^{2}\|e\|^{2}
\end{aligned}
$$

where $\left(Q_{0}^{1}\right)^{*}$ is the adjoint operator of $Q_{0}^{1}$. Hence, $\left(0 \wedge e_{m}\right)$ is a Bessel sequence. By Theorem 2 and Proposition 7, $Q_{0}^{1}$ is a topological isomorphism. Consider

$$
\begin{aligned}
\|e\|^{2}=\left\|Q_{0}^{1}\left(Q_{0}^{1}\right)^{-1} e\right\|^{2} & =\left\|\left(\left(Q_{0}^{1}\right)^{-1}\right)^{*}\left(Q_{0}^{1}\right)^{*} e\right\|^{2} \\
& \leq\left\|\left(\left(Q_{0}^{1}\right)^{-1}\right)^{*}\right\|^{2}\left\|\left(Q_{0}^{1}\right)^{*} e\right\|^{2} \\
& \leq \frac{\left\|\left(Q_{0}^{1}\right)^{-1}\right\|^{2}}{A} \sum_{m=1}^{\infty}\left|<\left(Q_{0}^{1}\right)^{*}(e), e_{m}>\right|^{2} \\
& \leq \frac{\left\|\left(Q_{0}^{1}\right)^{-1}\right\|^{2}}{A} \sum_{m=1}^{\infty}\left|<e, Q_{0}^{1}\left(e_{m}\right)>\right|^{2} .
\end{aligned}
$$

Consequently

$$
A\left\|\left(Q_{0}^{1}\right)^{-1}\right\|^{-2}\|e\|^{2} \leq \sum_{m=1}^{\infty}\left|<e, Q_{0}^{1}\left(e_{m}\right)>\right|^{2}
$$

Example 5. Let us consider any two-dimensional Hilbert space $V$. If $\left(e_{1}, e_{2}\right)$ is an orthonormal basis, it is an easy exercise to prove that $\left(e_{1}, e_{2}, e_{1}+e_{2}\right)$ is a frame. Consequently, the set $\left(0 \wedge e_{1}, 0 \wedge e_{2}, 0 \wedge\left(e_{1}+e_{2}\right)\right)$ is also a frame of $V$, if the conditions on the constants are satisfied. 
Corollary 1. If $\left(e_{m}\right)$ is a Bessel sequence in $E$ with bound $B$, then $\left(0 \wedge e_{m}\right)$ is also a Bessel sequence with the bound $B\left\|Q_{0}^{1}\right\|^{2}$.

Proof. The approach given in the first half of the proof of Proposition 17 provides the result.

Remark 1. Similarly for $Q_{0}^{2}$, if $c<1$ or $k<1$ and $\left(e_{m}\right)$ is a frame for $E$ with frame bounds $A, B$, then $\left(e_{m} \wedge 0\right)$ is a frame for $E$ with frame bounds $A\left\|\left(Q_{0}^{2}\right)^{-1}\right\|^{-2}, B\left\|Q_{0}^{2}\right\|^{2}$.

A frame $\left(e_{m}\right)$ is said to be an exact frame if for any $j \in \mathbb{N},\left(e_{m}\right)_{m \neq j}$ is not a frame.

Theorem 5 ([25]). Let $\left(e_{m}\right)$ be a frame for E. Then the following are equivalent:

- $\quad\left(e_{m}\right)$ is a Riesz basis for $E$;

- $\quad\left(e_{m}\right)$ is an exact frame;

- $\quad\left(e_{m}\right)$ has a biorthogonal sequence.

Proposition 18. If $c>1$ or $k^{\prime}<1$ and $\left(e_{m}\right)$ is an exact frame for $E$, then

- $\left(0 \wedge e_{m}\right)$ is an exact frame;

- $\quad\left(0 \wedge e_{m}\right)$ is a Riesz basis for E;

- $\quad\left(0 \wedge e_{m}\right)$ has a biorthogonal sequence.

Proof. Suppose $Q_{0}^{1}\left(e_{m}\right)$ is not an exact frame, then there exists $j \in \mathbb{N}$, such that $\left\{Q_{0}^{1}\left(e_{m}\right)\right\}_{m \neq j}$ is a frame. This gives $\left\{\left(Q_{0}^{1}\right)^{-1}\left(Q_{0}^{1}\left(e_{m}\right)\right)\right\}_{m \neq j}=\left(e_{m}\right)_{m \neq j}$ is also a frame, which is a contradiction to $\left(e_{m}\right)$ is an exact frame. Therefore, $\left(0 \wedge e_{m}\right)$ is an exact frame.

By Theorem $5,\left(0 \wedge e_{m}\right)$ is a Riesz basis for $E$, and it has a biorthogonal sequence.

Theorem 6 ([28]). If $\left(e_{m}\right)$ is a frame with frame bounds $A, B$ and $\left(e_{m}^{\prime}\right)$ satisfies

$$
R:=\sum_{m}\left\|e_{m}-e_{m}^{\prime}\right\|^{2}<A
$$

then $\left(e_{m}^{\prime}\right)$ is also a frame with frame bounds $A\left(1-\sqrt{\frac{R}{A}}\right)^{2}$ and $B\left(1+\sqrt{\frac{R}{B}}\right)^{2}$.

In the next set of results, we neglect the condition $c\|e \wedge 0\|=\|0 \wedge e\|$ and substitute it with a different hypothesis.

Theorem 7. Let $\left(e_{m}\right)$ be a frame with frame bounds $A, B>0$ in E. If there exists a sequence of real numbers $\left(c_{m}\right)$, such that

$$
c_{m}<1,\left\|0 \wedge e_{m}\right\|=c_{m}\left\|e_{m} \wedge 0\right\| \text { and } R=\sum_{m}\left(\frac{c_{m}}{1-c_{m}}\right)^{2}\left\|e_{m}\right\|^{2}<A,
$$

then $\left(e_{m} \wedge 0\right)$ is a frame with frame bounds $A\left(1-\sqrt{\frac{R}{A}}\right)^{2}$ and $B\left(1+\sqrt{\frac{R}{B}}\right)^{2}$ in $E$.

Proof. From the given hypothesis and (9), we have

$$
\left\|e_{m} \wedge 0-e_{m}\right\|=c_{m}\left\|e_{m} \wedge 0\right\|
$$

This implies

$$
\left\|e_{m} \wedge 0-e_{m}\right\| \leq c_{m}\left\|e_{m} \wedge 0-e_{m}\right\|+c_{m}\left\|e_{m}\right\|,
$$

and therefore

$$
\left\|e_{m} \wedge 0-e_{m}\right\| \leq \frac{c_{m}}{1-c_{m}}\left\|e_{m}\right\| .
$$

Using Theorem 6, we conclude the proof. 
Example 6. Let us consider the trigonometric frame $\left(\tau_{m}\right)=\{1\} \cup(\cos (m t), \sin (m t))$ in $\mathcal{L}^{2}(2 \pi)$. Taking the scale factors of the fractal convolution defined as $c_{m}:=1 / m$, the conditions of the previous theorem are satisfied, and thus the system $\left(\tau_{m} \wedge f_{0}\right)$ is also a frame, where $f_{0}$ denotes the null function.

Remark 2. We can prove similar result for $Q_{0}^{1}$, that is, if $\left(e_{m}\right)$ is a frame and there exists $\left(c_{m}\right)$, such that

$$
c_{m}<1,\left\|0 \wedge e_{m}\right\|=c_{m}\left\|e_{m} \wedge 0\right\| \text { and } R=\sum_{m}\left(\frac{1}{1-c_{m}}\right)^{2}\left\|e_{m}\right\|^{2}<A,
$$

then $\left(0 \wedge e_{m}\right)$ is also a frame in $E$.

Remark 3. Similarly if $\left(e_{m}\right)$ is a frame with frame bounds $A, B>0$, in $E$ and $R_{1}=\sum_{m} k^{2}\left\|e_{m}\right\|^{2}<A$ (or) $R_{2}=\sum_{m}\left(k^{\prime}\right)^{2}\left\|e_{m}\right\|^{2}<A$, then $\left(e_{m} \wedge 0\right)$ (or) $\left(0 \wedge e_{m}\right)$ is a frame with frame bounds $A\left(1-\sqrt{\frac{R_{1}}{A}}\right)^{2}, B\left(1+\sqrt{\frac{R_{1}}{B}}\right)^{2}$ (or) $A\left(1-\sqrt{\frac{R_{2}}{A}}\right)^{2}, B\left(1+\sqrt{\frac{R_{2}}{B}}\right)^{2}$, respectively, in E. Because due to the properties of the convolution

$$
\left\|e_{m} \wedge 0-e_{m}\right\|=\left\|e_{m} \wedge 0-e_{m} \wedge e_{m}\right\| \leq k\left\|e_{m}\right\|
$$

and

$$
\left\|0 \wedge e_{m}-e_{m}\right\|=\left\|0 \wedge e_{m}-e_{m} \wedge e_{m}\right\| \leq k^{\prime}\left\|e_{m}\right\| .
$$

\section{Conclusions and Potential Applications}

In this present work, we consider binary operations in metric spaces satisfying two inequalities related to the metric. Remarkable particular cases are the logical conjunction and disjunction, the union of compact sets and the fractal convolution of functions that we proposed in previous papers. This correspondence propitiates two partial or side operators on the space. Some properties of these maps are derived, along with additional results concerning the operation on subsets. For instance, a convolution naturally induces a similar operation on the set of compact sets of the space. In the case of a normed linear space, inspired by the fractal convolution, we work with the assumption that the associated operator is linear. Consequently, the side or partial convolutions with zero are also linear. This fact enables the definition of convolved Schauder bases of the space. At the end, the side convolution operators are studied in the framework of Hilbert spaces, and the existence of convolved Riesz and Bessel sequences and frames is proven.

The proposed theory, that generalizes the fractal case, may be useful in communication theory, signal analysis, operator theory, functional and harmonic analysis, and general study of metric spaces. A large number of problems of applied science must be approached by means of Approximation Theory. In particular, the approximation and interpolation of sets of data play a crucial role in all the social and scientific fields. In many cases, the approximative function is constructed as a linear combination of elements chosen from a suitable system. As described in Sections 4 and 5, the convolution provides additional bases and frames in the considered spaces. This fact enables the selection of an appropriate spanning family for a specific case. For instance, one can define bases of functions whose graphs own non-integer fractal dimensions beginning from very smooth maps as polynomial, trigonometric, etc. For irregular signals, these fractal functions may be more suitable than the classical. A standard function cannot approximate properly a chaotic signal possessing a self-similar graph. In this way, the convolution enlarges the field of Approximation and Functional Theory. For general metric spaces, convolution provides elements to a given distance of the components. If this quantity is small, the convolved elements can be considered a perturbed version of the originals. In this case, the new elements are close but at the same time may possess different properties, thus providing a wider spectrum of possibilities for the optimization of a given phenomenon. 


\begin{abstract}
Author Contributions: Conceptualization, M.A.N. and P.R.; methodology, M.A.N. and P.R.; validation, M.A.N. and A.K.B.C.; formal analysis, P.R.; writing-original draft preparation, M.A.N.; writing-review and editing, P.R. and A.K.B.C.; supervision, M.A.N. and A.K.B.C. All authors have read and agreed to the published version of the manuscript.
\end{abstract}

Funding: This research received no external funding.

Institutional Review Board Statement: Not applicable.

Informed Consent Statement: Not applicable.

Data Availability Statement: Not applicable.

Acknowledgments: The authors are grateful to the anonymous referees for wide-ranging comments and constructive suggestions that improved the presentation of the paper.

Conflicts of Interest: The authors declare no conflict of interest.

\title{
References
}

1. Fréchet, M.R. Sur Quelque Points du Calcul Fonctionnel. Rendic. Circ. Mat. Palermo 1906, 22 , 1-74. [CrossRef]

2. Byrant, V. Metric Spaces: Iteration and Application; Cambridge University Press: New York, NY, USA, 1985.

3. Heinonen, J. Lectures on Analysis on Metric Spaces; Springer: New York, NY, USA, 2001.

4. Lahti, P. Discrete convolutions of BV functions in quasiopen sets in metric spaces. Calc. Var. Partial. Differ. Equ. 2020, 59, 27. [CrossRef]

5. Pap, E.; Štajner, I. Generalized pseudo-convolution in the theory of probabilistic metric spaces, information, fuzzy numbers, optimization, system theory. Fuzzy Sets Syst. 1999, 102, 393-415. [CrossRef]

6. Navascués, M.A. Fractal functions of discontinuous approximation. J. Basic Appl. Sci. 2014, 10, 173-176. [CrossRef]

7. Navascués, M.A.; Massopust, P. Fractal convolution: A new operation between functions. Fract. Calc. Appl. Anal. 2019, 22, 619-643. [CrossRef]

8. Veisi, A.; Delbaznasab, A. Metric spaces related to Abelian groups. Appl. Gen. Top. 2021, 22, 169-181. [CrossRef]

9. Karami, A.; Shakery, R.; Sedghi, S.; Altun, I. Coupled fixed point results on metric spaces defined by binary operations. Carpathian Math. Publ. 2018, 10, 313-323. [CrossRef]

10. Schweizer, B.; Sklar, A. Statistical metric spaces. Pac. J. Math. 1960, 10, 314-334. [CrossRef]

11. Ziaie, S.A.; Ray, S.; Mashinchi, M.; Kamram, R. Boolean structure of triangular norms. Mathw. Soft Comput. 1999, 6, 63-78.

12. Conant, G. Distance structures for generalized metric spaces. Ann. Pure Appl. Log. 2017, 168, 622-650. [CrossRef]

13. Halmos, P.; Givant, S. Introduction to Boolean Algebras; Springer: New York, NY, USA, 2009.

14. Barnsley, M.F. Fractals Everywhere; Dover Publications: Mineola, NY, USA, 2012.

15. Chand, A.K.B.; Vijender, N.; Navascués, M.A. Shape preservation of scientific data through rational fractal splines. Calcolo 2014, 51, 329-362. [CrossRef]

16. Mandelbrot, B.B. The Fractal Geometry of Nature; Freeman: New York, NY, USA, 1982

17. Manousopoulos, P.; Drakopoulos, V.; Theoharis, T. Curve fitting by fractal interpolation. In Transactions on Computational Science; Lecture Notes in Computer Science Book Series; Springer: Berlin, Germany, 2008; Volume 4750; pp. 85-103.

18. Manousopoulos, P.; Drakopoulos, V.; Theoharis, T. Parameter identification of 1D recurrent fractal interpolation functions with applications to imaging and signal processing. J. Math. Imaging Vis. 2011, 40, 162-170. [CrossRef]

19. Navascués, M.A.; Chand, A.K.B. Fundamental sets of fractal functions. Acta Appl. Math. 2008, 100, 247-261. [CrossRef]

20. Banach, S. Sur les opérations dans les ensembles abstraits et leur application aux équations intégrales. Fundam. Math. 1922, 3, 133-181. [CrossRef]

21. Górniewicz, L. Topological Fixed Point Theory of Multivalued Mappings; Kluwer: Dordrecht, The Netherlands, 1999.

22. Secelean, N.A. Approximation of the attractor of a countable iterated function system. Gen. Math. 2009, 17, $221-231$.

23. Casazza, P.G.; Christensen, O. Perturbation of operators and applications to frame theory. J. Fourier Anal. Appl. 1997, 3, 543-557. [CrossRef]

24. Navascués, M.A.; Viswanathan, P.; Mohapatra, R.N. Convolved fractal bases and frames. Adv. Oper. Theory 2021, 6, 1-23. [CrossRef]

25. Christensen, O. Frames and Bases: An Introductory Course; Birkhauser: Boston, MA, USA, 2008.

26. Singer, I. Bases in Banach Spaces I; Springer: New York, NY, USA, 1970.

27. Haar, A. Zur Theorie der orthogonalen Funktionensysteme. Math. Ann. 1910, 69, 331-371. [CrossRef]

28. Christensen, O. Frame perturbations. Proc. Am. Math. Soc. 1995, 123, 1217-1220. [CrossRef] 